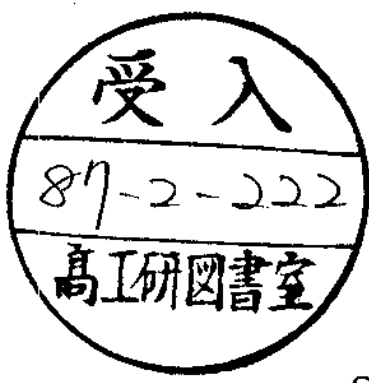

\title{
SUPERSYMMETRY BREAKING IN THE OBSERVABLE SECTOR OF SUPERSTRING MODELS
}

\author{
John Ellis, A.B. Lahanas*), D.V. Nanopoulos ${ }^{* *)}$, M. Quirós ${ }^{\times)}$and F. Zwirner ${ }^{\times \times)}$ \\ CERN, Geneva
}

\begin{abstract}
We discuss the communication of supersymmetry breaking from the gravitino mass $\mathrm{m}_{3 / 2}$ to gaugino masses $\mathrm{m}_{1 / 2}$, scalar masses $m_{0}$, and trilinear scalar couplings $A$ in superstring models, assuming that the effective low-energy $\mathrm{N}=1, \mathrm{~d}=4$ supergravity has a modified 'no-scale' structure. It is known that $\mathrm{m}_{1 / 2} \neq 0$ at the one-loop level, whilst squared scalar masses $m_{0}^{2}$ vanish at the one-loop level. We analyse a large class of two-loop diagrams, showing that their quadratic divergences cancel and that they contribute $\mathrm{m}_{0}^{2}=\mathrm{O}\left(\mathrm{m}_{3 / 2}^{4} / \mathrm{m}_{\mathrm{P}}^{2}\right)$. We also show that $\mathrm{A}$ appears in one-loop order, but is much smaller than $m_{1 / 2}$. Therefore the initial conditions $m_{1 / 2} \gg A \gg m_{0}$ are appropriate in renormalization group analyses of effective low-energy modeis inspired by the superstring.
\end{abstract}

*) Permanent address: Nuclear and Particle Physics Section, Physics Department, University of Athens, Athens 157-71, Greece.

**) Present address: Physics Department, University of Wisconsin, Madison, WI 53706, USA.

x) Permanent address: Instituto de Estructura de la Materia, Madrid, Spain.

$\times x$ ) Present address: Lawrence Berkeley Laboratory, Berkeley, CA 94720, USA. Also at International School for Advanced Studies, Trieste and Istituto Nazionale di Fisica Nucleare, Padua, Italy. 
One of the key issues in the extraction of phenomenology [1] from the superstring [2] is to determine how supersymmetry is broken. A plausible suggestion $[3,4]$ is that this is triggered by gaugino condensation in the hidden sector which generates a non-zero gravitino mass $m_{3 / 2}$. In order for the cosmological constant to vanish at the tree level, this condensate must cohabit with some other condensate, whose origin is more obscure but may be the antisymmetric tensor field $\mathrm{H}_{\mu \nu \mathrm{Q}}$ [4]. The next question is, How is this supersymmetry breaking is communicated to the observable sector? Here there are three possible classes of soft supersymmetry-breaking parameters, namely gaugino masses $m_{1 / 2}$, scalar masses $m_{0}$, and trilinear scalar couplings A. None of these [5] are present at the tree level when supersymmetry is broken spontaneously in the hidden sector as described above. One expects these supersymmetry-breaking parameters to be generated by loop corrections, so the next questions are, How many loops? which loops? which of $\mathrm{m}_{1 / 2}, \mathrm{~m}_{0}$, and $\mathrm{A}$ is the greater? and how large are the resulting supersymmetry-breaking parameters relative to $m_{3 / 2}$ ?

These questions are analysed here using the effective low energy $\mathrm{N}=1$ supergravity theory obtained by truncation from manifold compactification of the superstring [6], which is of a modified no-scale form [7]. It contains two relevant gauge singlet fields $S$ and $T$ as well as the gauge non-singlet fields $\phi^{\mathrm{i}}$, and has the following Kähler potential $\mathcal{G}$ and gauge kinetic terms:

$$
\begin{aligned}
& y=\varphi_{0}-\ln |w|^{2}: \varphi_{0}=\ln \left(S+S^{t}\right)+3 \ln \left(T+T^{t}-2 \phi_{i}^{t} \phi^{i}\right)(1 a) \\
& f_{a b}=S_{a b} S
\end{aligned}
$$

where $\mathrm{W}\left(\mathrm{S}, \phi^{\mathrm{i}}\right)$ is the superpotential which takes the form

$$
W\left(S, \phi^{i}\right)=\lambda_{i j k} \phi^{i} \phi^{j} \phi^{k}+\Omega(s): \Omega(s)=c+h \exp (-k s)(2)
$$

with $\lambda_{\mathrm{ijk}}$ being the Yukawa couplings in the gauge non-singlet sector, $\mathrm{c}$ and $\mathrm{h}$ result from $\mathrm{H}_{\mu \nu_{\ell}}$ and gaugino condensation, respectively and $k=8 \pi^{2} / c_{2}\left(G^{\prime}\right)$ where $G^{\prime}$ is the subgroup of É whose gauginos condense. It has been argued previously [8] that $\mathrm{m}_{0}$ and $\mathrm{A}$ vanish at the one-loop level, and to all orders [9] when the gauge couplings of the $\phi^{i}$ are neglected, if one works with the minimal no-scale model corresponding to the $\left(\mathrm{T}, \phi^{\mathrm{i}}\right)$ sector of the Kähler potential (1a). In this paper we study to what extent these results may [10] be modified by the inclusion of the $S$ field in the model (1). It is known $[11,12]$ that $m_{/ 2}$ is non-zero at the one-loop level in the model $(1)$, and we will compare the magnitudes of $\mathrm{m}_{1 / 2}, \mathrm{~m}_{0}$, and $\mathrm{A}$.

We find that two-loop diagrams may generate a non-zero value of $m_{0}$ which is at most of order

$$
m_{0} \approx \sqrt{\frac{\lambda^{2}}{16 \pi^{2}} \frac{\alpha}{\pi}} m_{3 / 2 / m_{p}}^{2}
$$

in the phenomenologically relevant limit $\mathrm{m}_{3 / 2} \ll \mathrm{m}_{\mathrm{P}}$. This is much smaller than the observable sector gaugino mass which we [12] have estimated to be of order 


$$
m_{1 / 2} \approx\left(\frac{\alpha}{\pi}\right)^{4 / 3} m_{3 / 2}^{5 / 3} / m_{p}^{2 / 3}
$$

We also find that the trilinear supersymmetry-breaking parameter $\mathrm{A}$ is expected to be small:

$$
A=\hat{A} m_{1 / 2}: \hat{A}=-\frac{9}{12 \sqrt{3}}
$$

where $\alpha \equiv \mathrm{g}^{2} / 4 \pi$. Both (3) and (4) result from the shift in $\langle 0|\mathrm{~S}| 0\rangle$ which is induced by one-loop corrections to the effective potential. The results (3)-(5) should be interpreted as initial conditions for a renormalization group analysis of the effective low-energy theory. Although $m_{1 / 2} \gg m_{0}$ and $A$ initially, the physical values renormalized by gauge loops (not considered here) and chiral loops are of similar magnitude. The results (3), (4), (5) contrast with those obtained from the most general supergravity model compatible with the classical symmetries of the string [13-15], and with those of the most general supergravity model compatible with the symmetries of quantum corrections in string theory $[16,15]$.

We first make a diagrammatic analysis of one-loop contributions to $m_{0}$ in the effective theory (1). This step provides no new results, but gives us a basis for the subsequent discussion of two-loop diagrams. The squared scalar mass is defined by

$$
\left.m_{o_{j}^{i}}^{2} \equiv \frac{\partial^{2} V}{\partial \phi_{i}^{+} \partial \phi^{j}}\right|_{\phi^{i}=0}
$$

and the contributing one-loop diagrams are shown in Fig. 1. We work in the harmonic gauge $\gamma^{\mu} \psi_{\mu}=0$ so that there is no mixing in any fermion propagator ${ }^{*}$. Note that no diagrams involving the Yukawa couplings $\lambda$ appear because there is no supersymmetry breaking for the $\phi^{\mathrm{i}}$ fields at the tree level. This means that the supersymmetry-breaking one-loop effective potential $\mathrm{V}_{1}$ can depend on $\phi^{\mathrm{i}}$ only through the combination $S_{0} \equiv 3 \ln \left(T+T^{\dagger}-2 \phi_{i}^{\dagger} \phi^{i}\right)[8,9]$. This point can be verified explicitly by comparing the diagrams of Fig. 1 with those of Fig. 2, which contribute to $\partial \mathrm{V} / \partial \mathrm{T}$. Diagrams (a), (b), (c), (d), and (e) clearly correspond directly, whilst diagrams (f) and (g) of Fig. 2 do not correspond to anything in Fig. 1. However, a simple calculation shows that this is not a problem because both of these diagrams vanish at zero external momentum, and hence do not contribute to the effective potential. Diagrams (a) to (e) are quadratically divergent, but there is a physical momentum cut-off provided by the scale $\Lambda_{c}$ of gaugino condensation. Since $m_{3 / 2} \approx \Lambda_{c}^{3} / \mathrm{m}_{\mathrm{P}}^{2} \ll \mathrm{m}_{\mathrm{P}}$, the quadratically divergent pieces of the diagrams $\propto \Lambda_{\mathrm{c}}^{2} \operatorname{Str} \mathfrak{N}^{2}$ are much larger than the logarithmically divergent pieces $\propto \operatorname{Str} \mathfrak{T}^{4} \ln \mathfrak{N}^{2} / \Lambda^{2}$, and so on. The fact that the diagrams (a) to (e) give an effective potential which depends only on $S_{0}$ means that

*) Note that the gravitino propagator in this gauge is not the same as the propagators given by van Nieuwenhuizen [17]. 


$$
\left.\frac{\partial^{2} V_{1}}{\partial \phi^{+} \partial \phi}\right|_{\phi=0} \propto \frac{\partial V_{1}}{\partial \xi_{0}} \propto \frac{\partial V_{1}}{\partial T} \propto S_{t r} \mu^{2} \Lambda^{2}
$$

In the true vacuum of the theory, we must have $\partial \mathrm{V} / \partial \mathrm{T}=0$ and we assume that the unknown parts of

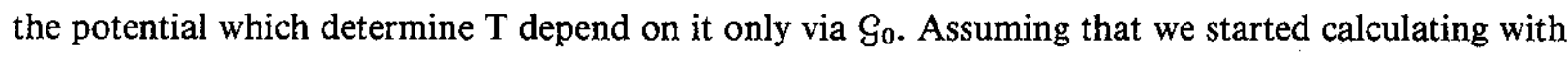
$\partial \mathrm{V}_{0} / \partial \mathrm{T}=0$ at the tree level, the non-zero value of $\partial \mathrm{V}_{1} / \partial \mathrm{T}(7)$ tells us that we must shift the vacuum expectation value of the $\mathrm{T}$ field. This can be taken into account diagrammatically by introducing counter terms $\mathrm{V}_{\mathrm{c}_{1}}$ depending on $\mathrm{So}_{0}$ which ensure that

$$
\frac{\partial V}{\partial T}=\frac{\partial}{\partial T}\left(V_{0}+V_{1}+V_{c_{1}}+O\left(\hbar^{2}\right)\right)=0
$$

However, it is then obvious from (7) that the counter terms also cancel $\partial^{2} \mathrm{~V}_{1} / \partial \phi^{\dagger} \partial \phi$ :

$$
\frac{\partial^{2} V}{\partial \phi^{+} \partial \phi}=\frac{\partial^{2}}{\partial \phi^{+} \partial \phi}\left(V_{0}+V_{1}+V_{c_{1}}\right)=O\left(\hbar^{2}\right)
$$

Thus any gauge non-singlet squared scalar mass can be at most a two-loop effect, which we now analyse.

The above argument that $\mathrm{m}_{0}^{2}=\partial^{2} \mathrm{~V} / \partial \phi^{\dagger} \partial \phi \propto \partial \mathrm{V} / \partial \mathrm{T}=0$ can be extended [9] to all loops in the minimal no-scale model in which one neglects $S$ and the Yukawa couplings $\lambda$. If one includes $\lambda$ but still neglects $S$, a similar argument can be made on the basis of a plausible conjecture for the form of the n-loop effective potential [9]. If one includes $S$ but neglects $\lambda$, it is easy to see that the first non-zero contribution to $\mathrm{m}_{0}^{2}$ is a three-loop effect:

$$
m_{0}=O(\hbar) m_{1 / 2}^{2}: m_{1 / 2}=O(\hbar)
$$

A full two-loop analysis of $m_{0}^{2}$ will be given elsewhere [18]. Here we concentrate on the most interesting two-loop diagrams, namely those which are $\propto \lambda^{2}$ and include S-superfield loops, shown in Fig. 3. One diagram of this type (see Fig. 3a) has been mentioned previously [10] as a possible source of $m_{0}^{2}$. It has been argued [12] that this particular diagram gives $m_{0}^{2} \leqslant m_{3 / 2}^{4} / m_{P}^{2}$, and we now show that this conclusion holds true for all the diagrams in Fig. 3.

We first introduce the following notation:

$$
\left.\begin{array}{c}
\left.a \equiv\left(\frac{1}{\Omega(s)} \frac{\partial \Omega}{\partial s}\right)^{2}\right|_{s=s_{0}},\left.G \equiv\left(\frac{1}{\Omega^{*}} \frac{\partial^{2} \Omega^{*}}{\partial s^{2}}\right)\right|_{s=s_{0}} \\
\left.\Omega_{0} \equiv(\Omega(s))\right|_{s=s_{0}}
\end{array}\right\}
$$


where $S_{0}$ is the tree-level expectation value of $S$. In terms of the quantities (11)

$$
m_{3 / 2}^{2}=e^{-\xi_{0}} \sqrt{a}\left|\Omega_{0}\right|^{2}
$$

where we have used the relation $\sqrt{\mathrm{a}}=\left(\mathrm{S}_{0}+\mathrm{S}_{0}^{\dagger}\right)^{-1}$ which is valid when the tree-level potential is minimized. For the fields in the $S$ supermultiplet we have

$$
m_{\tilde{s}}=m_{3 / 2} \frac{|G|}{a}, m_{s_{ \pm}}^{2}=m_{3 / 2}^{2}\left(1 \pm \frac{|G|}{a}\right)^{2}
$$

and the spin-zero $\mathrm{S}$ propagators are

$$
\left.\begin{array}{l}
P_{S^{+} S}(p)=\frac{i}{(2 \pi)}\left(\frac{1}{2 a}\right)\left(\frac{1}{p^{2}-m^{2}}+\frac{1}{p^{2}-m^{2}}\right) \\
P_{S S}(p)=\frac{i}{(2 \pi)^{4}}\left(\frac{e^{-2 i \theta}}{2 a}\right)\left(\frac{1}{p^{2}-m^{2}}-\frac{1}{p^{2}-m^{2}}\right)
\end{array}\right\}
$$

where the angle $\theta$ diagonalizes the spin-zero mass matrix. Using the Weyl representation, the $\widetilde{\mathrm{S}}$ fermion propagators take the form

$$
\left.\begin{array}{l}
(P \tilde{s} \overline{\tilde{s}})_{\alpha \dot{\beta}}=\frac{i}{(2 \pi)^{4}}\left(\frac{1}{2 a}\right) \frac{(\not)_{\alpha \beta}}{p^{2}-m_{\tilde{s}}^{2}}: \not p \equiv \sigma^{m} p_{\mu} \\
\left(P_{\tilde{s} \tilde{s})_{\alpha}^{\beta}=}=\frac{i}{(2 \pi)^{4}}\left(\frac{1}{2 a}\right) \frac{m_{\tilde{s}} \delta_{\alpha}^{\beta}}{p^{2}-m_{\tilde{\zeta}}^{2}}\right.
\end{array}\right\}
$$

The gauge non-singlet propagators are

$$
P_{\phi_{i}^{t} \phi^{j}}=\frac{i}{(2 \pi)^{4}}\left(\frac{e^{\rho_{0} / 3}}{6}\right) \frac{1}{p^{2}} \delta_{j}^{i}
$$

for spin zero, and

$$
\left(P_{\tilde{\phi} \bar{\phi}}\right)_{\alpha \dot{\beta}}=\frac{i}{(2 \pi)^{4}}\left(\frac{e^{g_{\alpha / 3}}}{12}\right) \frac{(\beta)_{\alpha \dot{\beta}}}{p^{2}}
$$

for spin half.

The table lists, for each of the diagrams (a) to (k) in Fig. 3, the contribution it makes to $\mathrm{m}_{0}^{2}$ and its quadratically divergent part. We use the notation 


$$
f \equiv \frac{1}{(2 \pi)^{8}} \frac{\lambda^{2}}{4} \frac{m_{3 / 2}^{2}}{\left(\Omega_{d}^{2}\right.}\left(\frac{e^{\zeta_{0 / 3}}}{6}\right)^{2}
$$

and denote the quadratically divergent parts of the one- and two-loop integrals which appear by

$$
Q_{0} \equiv Q \int \frac{d^{4} k}{k^{2}}, Q_{1} \equiv Q \int \frac{d^{4} k d^{4} q}{k^{2} q^{2}(k-q)^{2}}, Q_{2} \equiv Q \int \frac{d^{4} k d^{4} q}{k^{4} q^{2}}
$$

Here in the text we list the vertices which are needed to calculate each of the diagrams:

Fig.

$3 a$

$$
-\frac{m_{3 / 2}^{2}}{\sqrt{\Omega_{0}}} \frac{1}{\sqrt{a}}\left(G S^{t}+a S\right) \frac{\lambda \phi^{3}}{3 !}+\text { hic. }
$$

$3 b$

$$
-\frac{e^{-2 \xi_{\phi / 3}}}{4 !} \sqrt{a} \lambda^{2} \phi^{2} \phi^{2}-\frac{6}{a} m_{3 / 2}^{2} e^{-\xi_{\phi / 3}}\left|G S^{+}+a S\right|^{2} \phi \phi^{+}
$$

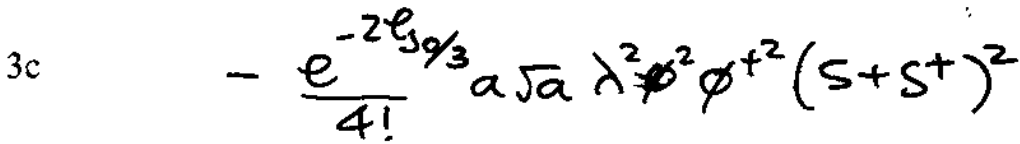

Bd

$$
-\frac{\lambda m_{3 / 2} \sqrt{a}}{\Omega_{0}} \phi^{2} \tilde{S} \tilde{\phi}+\text { hoc. }
$$

$3 e$

$$
-\frac{\lambda m_{3 / 2}}{\sqrt{2}_{0}} \sqrt{a}(\tilde{\phi} \tilde{\phi}) S_{\phi}+\text { hic. }
$$

$3 f$

$$
\begin{aligned}
& -\frac{e^{-2 \xi_{0 / 3}}}{4 !} \lambda^{2} \sqrt{a} \phi^{2} \phi^{t^{2}}-3|G| e^{-e_{0 / 3} m_{3 / 2}}(\tilde{s} \tilde{s}+\overline{\widetilde{s}} \overline{\widetilde{s}}) \phi \phi^{+} \\
& -3 i a e^{-e_{\phi / 3}}\left(\phi^{+} \overleftrightarrow{\partial}_{\mu} \phi\right) \frac{\tilde{\tilde{s}}}{\bar{\sigma}} \bar{\sigma}^{\mu} \tilde{s}
\end{aligned}
$$

$3 \mathrm{~g}$

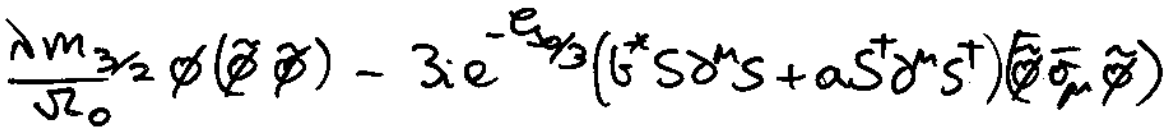

$$
\begin{aligned}
& + \text { hic. }
\end{aligned}
$$

$3 h$

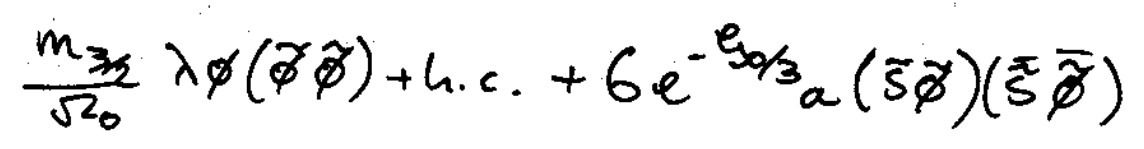

$3 i$

$$
\frac{\lambda m_{3 / 2} \phi(\not \partial \tilde{\phi})\left[1+\frac{1}{4}\left(4 a-b^{*}\right) s^{2}+\frac{b}{4} s^{t^{2}}+\frac{a}{2} s s^{+}\right]}{+ \text {hic. }}
$$

5 
$3 \mathrm{k}$

$$
\frac{\lambda^{2}}{4 !} \sqrt{a} e^{-2 \varphi_{0 / 3}}\left(S+S^{+}\right) \phi^{2} \phi^{t^{2}}
$$

It is immediately apparent from adding up the last column in the table that the quadratically divergent contributions to $\mathrm{m}_{0}^{2}$ which are $\propto \mathrm{m}_{\widetilde{\mathrm{s}}}^{2}$ cancel. There is a residual quadratic divergence $\propto \mathrm{m}_{3 / 2}^{2}$, but we expect this to cancel against other two-loop diagrams which do not involve S propagation. We are therefore left with the following estimate for the net two-loop contribution to $\mathrm{m}_{0}^{2}$ :

$$
m_{0}^{2}=O\left(\frac{g^{2} \lambda^{2}}{(2 \pi)^{4}} \frac{e^{-\varepsilon_{0}}}{288}\right) m_{3 / 2}^{4} / m_{p}^{2}
$$

where we have used

$$
\frac{m_{3 / 2}^{2}}{\left|\Omega_{0}\right|^{2}}=\frac{e^{-\xi_{0}}}{2 S_{R}}=\frac{g^{2}}{2} e^{-e_{0}}: S_{R} \equiv \operatorname{ReS}
$$

We apologize for the fact that so far we lack the courage to evaluate the coefficient of the $\mathrm{O}\left(\mathrm{m}_{3 / 2}^{4}\right)$ contribution (21).

Equation (21) is the main result of this paper. As mentioned above, we do not expect [9] a quadratically divergent contribution to $\mathrm{m}_{0}^{2}$ from diagrams which do not involve $\mathrm{S}$ propagators. In a future publication [18] we will present a complete analysis of the two-loop diagrams which are $\propto \lambda^{2}$ and only involve particles from the $\left(T, \phi^{i}\right)$ supermultiplets. As a sneak preview, we draw the reader's attention to the class of such diagrams shown in Fig. 4. They, together with Figs. $3 \mathrm{~b}$ and $3 \mathrm{f}$, do have a net quadratic divergence $\propto \mathrm{m}_{3 / 2}^{2}$, but it is solely due to the one-loop subgraphs shown in Fig. 1 . When we include the one-loop counter term which ensures $\partial \mathrm{V} / \partial \mathrm{T}=\mathrm{O}\left(\hbar^{2}\right)$ as in Eq. (8), this net quadratic divergence is cancelled, and this class of diagrams also contributes at $\operatorname{most} O\left(\mathrm{~m}_{3 / 2}^{4} / \mathrm{m}_{\mathrm{P}}^{2}\right)$ to $\mathrm{m}_{0}^{2}$.

We conclude with a final comment on the trilinear supersymmetry-breaking parameter $\mathrm{A}$. This acquires a one-loop contribution from the same shift in $\langle 0|\mathrm{~S}| 0\rangle$ which contributed [12] to $\mathrm{m}_{1 / 2}$ in $O(h)$. A simple calculation gives

$$
\begin{array}{r}
A_{N S}=\frac{1}{2 S_{R}} \frac{1}{\left(2 T_{R}\right)^{3}}\left\langle 0\left|\Omega(S)-2 S_{R} \frac{\partial \Omega}{\partial S}\right| 0\right\rangle \\
\left(T_{R} \equiv \operatorname{ReT}\right)
\end{array}
$$

using the non-standard normalization of the $\phi$ fields corresponding to the $\phi^{\mathrm{i}}$ propagators (16) and (17), where 


$$
m_{1 / 2}=\frac{1}{\left(2 S_{R}\right)^{1 / 2}} \frac{1}{\left(2 T_{R}\right)^{3 / 2}}\left\langle 0\left|\Omega(s)-2 S_{R} \frac{\partial \Omega}{\partial S}\right| 0\right\rangle
$$

Comparing (23) and (24) we find

$$
A_{N S}=\frac{1}{\left(2 S_{R}\right)^{1 / 2}} \frac{1}{\left(2 T_{R}\right)^{3 / 2}} m_{1 / 2}
$$

Introducing the conventional normalization $\hat{\phi} \equiv\left(3 / T_{R}\right)^{1 / 2} \phi$ and the reduced coefficient $\bar{A} \equiv A / m_{1 / 2}$, we obtain

$$
\bar{A}=\frac{A_{N S}}{m_{1 / 2}}\left(\frac{T_{R}}{3}\right)^{3 / 2}=-\frac{9}{12 \sqrt{3}}
$$

which yields $\overline{\mathrm{A}} \approx 0.05$ for a plausible value $\mathrm{g}=\mathrm{O}(1)$.

Comparing the results (21) and (26) with our previous estimate (4) of $m_{1 / 2}$, it seems to us that the appropriate initial conditions to be used in renormalization group analyses of the effective low-energy theories [Eqs. (1)] inspired by the superstring that we have considered are:

$$
m_{1 / 2} \gg A \gg m_{0}
$$

given the phenomenologically necessary assumption $\mathrm{m}_{3 / 2} \ll \mathrm{m}_{\mathrm{P}}$. It is possible that other two-loop diagrams could yield a larger contribution $\mathrm{O}\left(\mathrm{m}_{3 / 2}^{2} \lambda_{\mathrm{c}}^{2}\right)$ to $\mathrm{m}_{0}^{2}$, or that higher-loop diagrams could make a contribution of this order. We find this unlikely, but the hierarchy (27) of initial conditions might even survive such an effect, because of the small coefficients (21) characteristic of multi-loop diagrams.

\section{Acknowledgements}

One of us (J.E.) thanks the Democritos Nuclear Research Centre for its kind hospitality while this work was completed.

The work of D.V.N. was supported in part by DOE grant DE-ACO2-76ER0081 and in part by the University of Wisconsin Research Committee with funds granted by the Wisconsin Alumni Research Foundation. The work of F.Z. is supported by a Fellowship of the Istituto Nazionale di Fisica Nucleare, Italy. 


\section{REFERENCES}

[1] For reviews, see J. Ellis, CERN preprint TH.4439/86 (1986);

H.-P. Nilles, CERN preprint TH.4444/86 (1986);

L.E. Ibáñez, CERN preprint TH.4459/86 (1986).

[2] J.H. Schwarz (ed.), Superstrings - the first 15 years (World Scientific, Singapore, 1985).

[3] J.-P. Derendinger, L.E. Ibáñez and H.-P. Nilles, Phys. Lett. 155B, 65 (1985).

[4] M. Dine, R. Rohm, N. Seiberg and E. Witten, Phys. Lett. 156B, 55 (1985).

[5] J. Ellis, C. Kounnas and D.V. Nanopoulos, Nucl. Phys. B247, 373 (1984).

[6] E. Witten, Phys. Lett. 155B, 151 (1985).

[7] E. Cremmer, S. Ferrara, C. Kounnas and D.V. Nanopoulos, Phys. Lett. 133B, 61 (1983);

J. Ellis, A.B. Lahanas, D.V. Nanopoulos and K. Tamvakis, Phys. Lett. 134B, 429 (1984);

J. Ellis, C. Kounnas and D.V. Nanopoulos, Nucl. Phys. B241, 406 (1984).

[8] J.D. Breit, B.A. Ovrut and G. Segré, Phys. Lett. 162B, 303 (1985);

P. Binétruy and M.K. Gaillard, Phys. Lett. 168B, 347 (1986).

[9] G. Diamandis, J. Ellis, A.B. Lahanas and D.V. Nanopoulos, Phys. Lett. 173B, 303 (1986).

[10] Y.J. Ahn and J.D. Breit, Nucl. Phys. B273, 75 (1986);

Y.J. Ahn and G. Segré, private communication.

[11] P. Binétruy, S. Dawson and I. Hinchliffe, Phys. Lett. 179B, 262 (1986) and Berkeley preprint LBL-22322 (1986).

[12] J. Ellis, D.V. Nanopoulos, M. Quirós and F. Zwirner, Phys. Lett. 180B, 83 (1986).

[13] C. Burgess, A. Font and F. Quevedo, Nucl. Phys. B272, 661 (1986).

[14] A. Font, F. Quevedo and M. Quirós, CERN preprint TH.4577/86 (1986).

[15] S. Ferrara, A. Font, F. Quevedo, M. Quirós and M. Villasante, CERN preprint TH.4580/86 (1986).

[16] L.E. Ibáñez and H.-P. Nilles, Phys. Lett. 169B, 354 (1986);

H.-P. Nilles, Phys. Lett. 180B, 240 (1986).

[17] P. van Nieuwenhuizen, Phys. Rep. 68C, 189 (1981).

[18] J. Ellis, A.B. Lahanas, D.V. Nanopoulos, M. Quirós and F. Zwirner, in preparation (1987). 


\begin{tabular}{|c|c|c|}
\hline Diagram & Contribution to $\mathrm{m}_{0}^{2}$ & $\begin{array}{l}\text { Quadratically } \\
\text { divergent part }\end{array}$ \\
\hline (a) & $\begin{array}{l}-f m_{3 / 2}^{2}\left\{\left(1+\frac{\mid b^{2}}{a^{2}}\right) \int \frac{d^{4} k d^{4} q}{k^{2}(k-q)^{2}}\left[\frac{1}{q^{2}-m^{2}}+\frac{1}{q^{2}-m^{2}}\right]\right. \\
\left.+\left(\frac{b e^{2 i \theta}}{a}+h \cdot c\right) \int \frac{d^{4} k d^{4} q}{k^{2}(k-q)^{2}}\left[\frac{1}{q^{2}-m_{+}^{2}}-\frac{1}{q^{2}-m^{2}}\right]\right\}\end{array}$ & $-2 f\left(m_{: 3}^{2}+m_{3 / 2}^{2}\right) Q_{1}$ \\
\hline$(G)$ & $\begin{aligned}-2 f m_{3 / 2}^{2} & \left\{\left(1+\frac{\left|G^{2}\right|^{2}}{a^{2}}\right) \int \frac{d^{4} k d^{4} q}{k^{4}}\left[\frac{1}{q^{2}-m^{2}}+\frac{1}{q^{2} \cdot m_{2}^{2}}\right]\right. \\
& \left.+\left(\frac{b e^{2 i \theta}}{a}+h \cdot c \cdot\right) \int \frac{d^{4} k d^{4} q}{k^{2}\left(k-q^{2}\right.}\left[\frac{1}{q^{2}-m^{2}}+\frac{1}{q^{2}-m^{2}}\right]\right\}\end{aligned}$ & $-4 f\left(m_{\xi}^{2}+m_{3 / 2}^{2}\right) Q_{2}$ \\
\hline (c) & $\begin{array}{l}-4 f\left\{\int \frac{d^{4} k d^{4} q}{R^{2}}\left[\frac{1}{q^{2}-m_{+}^{2}}+\frac{1}{q^{2}-m_{2}^{2}}\right]\right. \\
\left.\quad+\left(\frac{e^{2 i \theta}}{2}+\text { h.c. }\right) \int \frac{d^{4} k d^{4} q}{k^{2}}\left[\frac{1}{q^{2}-m_{+}^{2}}-\frac{1}{q^{2}-m_{2}^{2}}\right]\right\}\end{array}$ & $-8 f\left(m_{\tilde{S}}^{2}+m_{3 / 2}^{2}\right) Q_{2}$ \\
\hline (d) & $8 f \int \frac{d^{4} k d^{4} q k \cdot q}{k^{2}(k-q)^{2}\left(q^{2}-m_{\xi}^{2}\right)}$ & $4 f m_{\tilde{s}}^{2} Q_{1}$ \\
\hline (e) & $2 f \int \frac{d^{4} k d^{4} q k \cdot(k-q)}{k^{2}(k-q)^{2}}\left[\frac{1}{q^{2}-m_{+}^{2}}+\frac{1}{q^{2}-m^{2}-}\right]$ & $4 f\left(m_{3}^{2}+m_{3 / 2}^{2}\right)\left(Q_{2}-\frac{1}{2} Q_{1}\right)$ \\
\hline (f) & $-4 f\left\{\int \frac{d^{4} k d^{4} q k \cdot q}{k^{4}\left(q^{2}-m_{\xi}^{2}\right)}-m_{\tilde{s}}^{2} \int \frac{d^{4} k d^{4} q}{k^{4}\left(q^{2}-m_{\tilde{s}}^{2}\right)}\right\}$ & $4 f m_{\tilde{S}}^{2} Q_{2}$ \\
\hline (9) & $\begin{aligned}-2 f\{ & \left\{\int \frac{d^{+k} k d^{4} q k \cdot q}{k^{4}}\left[\frac{1}{q^{2}-m_{+}^{2}}+\frac{1}{q^{2}-m^{2}}\right]\right. \\
& \left.+\left(\frac{b e^{2 i \theta}}{a}+h \cdot c .\right) \int \frac{d^{4} k d^{4} q k \cdot q}{k^{4}}\left[\frac{1}{a^{2}-m_{+}^{2}}-\frac{1}{q^{2}-m_{-}^{2}}\right]\right\}\end{aligned}$ & 0 \\
\hline (h) & $2 f \int \frac{d^{4} k d^{4} q k \cdot q}{k^{4}\left(q^{2}-m_{\tilde{s}}^{2}\right)}$ & 0 \\
\hline (i) & $\begin{array}{l}-4 f\left\{\int \frac{d^{4} k d^{4} q}{k^{2}}\left[\frac{1}{q^{2}-m_{+}^{2}}+\frac{1}{q^{2}-m^{2}}\right]\right. \\
\left.\quad+\left(\frac{e^{2 i \theta}}{2}+h \cdot c \cdot\right) \int \frac{d^{4} k d^{4} q}{R^{2}}\left[\frac{1}{q^{2}-m_{+}^{2}}-\frac{1}{q^{2}-m^{2}}\right]\right\}\end{array}$ & $4 f\left(m_{\tilde{S}}^{2}+m_{3 / 2}^{2}\right) \theta_{2}$ \\
\hline (j) & $-4 f\left(v+v^{*}\right) \int \frac{d^{4} k}{k^{2}}: v \equiv\langle 0|s| 0\rangle_{1-100 p}$ & $-4 f\left(v+v^{*}\right) \theta_{0}$ \\
\hline (k) & $+4 f\left(v+v^{*}\right) \int \frac{d^{4} k}{k^{2}}$ & $+4 f\left(v+v^{*}\right) Q_{0}$ \\
\hline
\end{tabular}




\section{Figure captions}

Fig. 1 One-loop diagrams which might contribute to $\mathrm{m}_{0}^{2}$.

Fig. 2 One-loop contributions to $\partial V / \partial T$. Note the correspondence to diagrams (a) to (e) of Fig. 1.

Fig. 3 Two-loop diagrams which might contribute to $m_{0}^{2}$, selected because they (i) involve $S$ superfield propagation, (ii) are $\propto \lambda^{2}$.

Fig. 4 A subset of two-loop diagrams which might contribute to $m_{0}^{2}$ and are in one-to-one correspondence with (a), (b), (c), (f) and (g) of Fig. 1. 


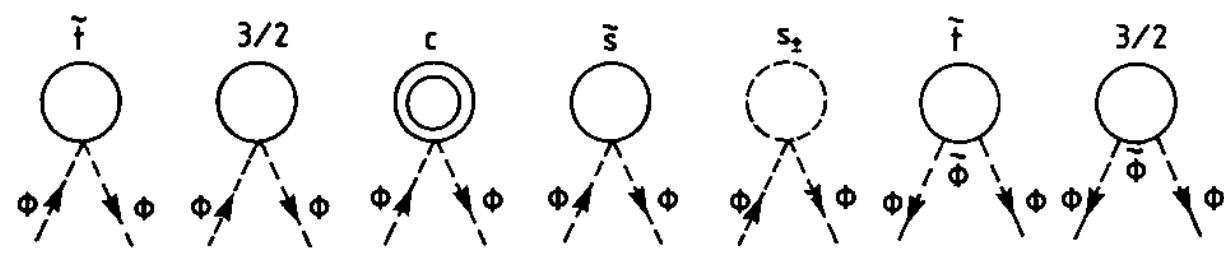
(a)
(b)
(c)
(d)
(e)
(f)
(g)

Fig. 1

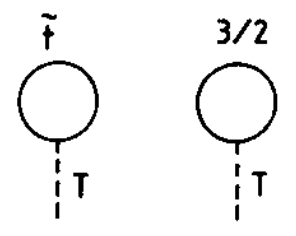

(a)

(b)<smiles>II(I)C1CCCC([Tl])CC1</smiles>

(d)

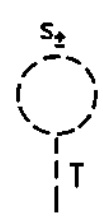

(e)

Fig. 2

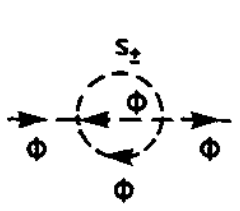

(a)

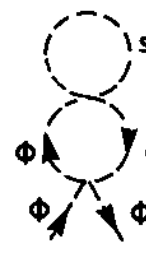

(b)

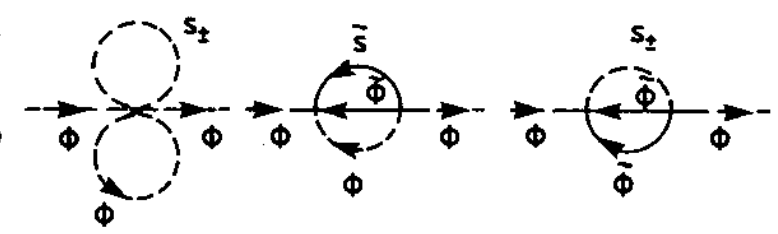

(c)

(d)

(e)

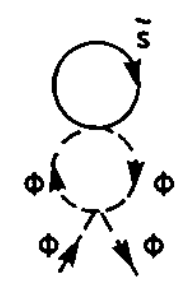

(f)

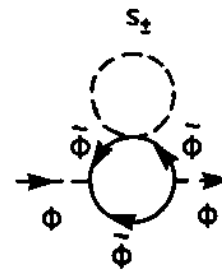

(g)

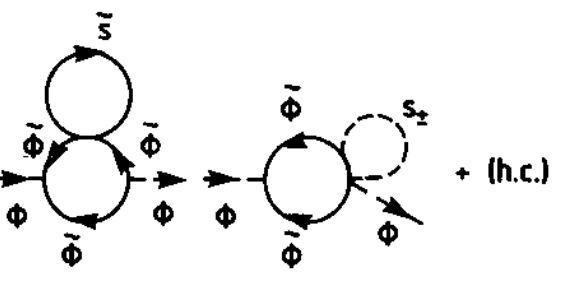

(h)

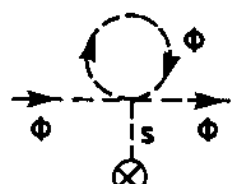

(k)

Fig. 3

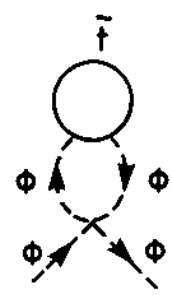

(a)

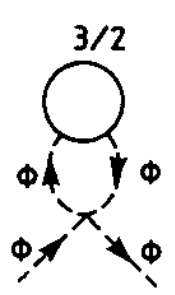

(b)

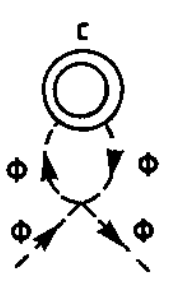

(c)

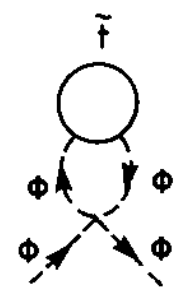

(d)

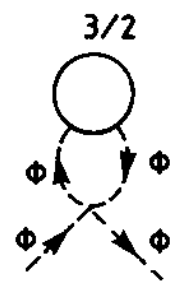

(e)

Fig. 4 